\title{
An innovative approach for migraine prevention in young age: a preliminary study
}

\author{
Susanna Usai • Licia Grazzi • Frank Andrasik • \\ Gennaro Bussone
}

(C) The Author(s) 2010. This article is published with open access at Springerlink.com

\begin{abstract}
Headache is one of the commonest conditions to affect children and adolescents in industrialized countries. Effective pharmacological treatments without side effects are still lacking. Ginkgolide B, an herbal constituent extract from ginkgo biloba tree leaves, is a natural antiplatelet activating factor (PAF). PAF is a potent proinflammatory and nociceptive agent released during the inflammation process. Therefore, Ginkgolide B can be considered a promising non-pharmacological tool for treatment of migraine with and without aura. We propose to determine the efficacy of Ginkgolide B as preventive treatment in a group of young patients suffering from migraine without aura. A small sample of 24 young patients suffering from migraine without aura entered the open-label prospective trial. Migraine without aura was diagnosed according to International Headache Society criteria. The treatment was well tolerated and the compliance was good. These preliminary data show that Ginkgolide B seems to be effective as preventive treatment in reducing migraine attack frequency and in attenuating the use of symptomatic medication in our small series of children with primary headache.
\end{abstract}

Keywords Headache - Young age - Ginkgolide B . Preventive treatment

S. Usai $(\bowtie) \cdot$ L. Grazzi · G. Bussone

Headache Center, National Neurological Institute "C. Besta",

Via Celoria, 11, 20133 Milan, Italy

e-mail: susanna.usai@istituto-besta.it

F. Andrasik

Department of Psychology, University of West Florida,

Pensacola, FL, USA

\section{Introduction}

Headache is one of the commonest conditions to affect children and adolescents in industrialized countries. Studies indicate a prevalence of 8 to $60 \%$ [1]. In over $40 \%$ of migraineurs the condition begins before 18 years of age [1]. Recurrent headaches turn out common in children and adolescents, and they often considerably reduce quality of life [2], with a negative impact on school and social activity [3]. Primarily, treatment of migraine in young age consists of avoidance of triggers, and regular habits of life (sleep, meals, computer\&TV, and sports). Very often parents prefer non-pharmacological treatment for their children, limiting the assumption of symptomatic medication when absolutely necessary for migraine attack. Treatment for the various forms of childhood headache has been the subject of wide debate in recent years. For migraine and tension-type headache, the same preventive drugs as used in adults are widely prescribed for children, but at reduced dosage. Effective pharmacological treatments without side effects are still lacking. Most of current non-pharmacological treatment modalities have been employed (either medication or behavioral) often with unsatisfactory results. Among non-pharmacological approach until now, magnesium has been successfully used for headache treatment in young patients, in particular for tension-type headache [4]. Few pharmacological studies have included treatment comparisons, one of the most recent experience has been performed by our group [5]. One of the most urgent and important problem with this kind of patients is to have therapeutic possibilities without side effects, which are common with pharmacological treatments.

Ginkgolide B, an herbal constituent extract from ginko biloba tree leaves, is a natural antiplatelet activating factor (PAF). PAF is a potent proinflammatory and nociceptive 
agent released during the inflammation process. In addition, Ginkgolide B modulates the action of glutamate acid, the main excitatory neurotransmitter of CNS. It is known that abnormal levels of glutamate may cause spreading depression and migraine aura in the susceptible individuals and the PAF, released from platelets and leukocytes during the first phase of migraine without aura attacks, sensitizes the trigeminal-vascular endings and induces pain. Therefore, Ginkgolide B can be considered a promising nonpharmacological tool for treatment of migraine with and without aura [6].

On the basis of this evidence, we propose to determine the efficacy of Ginkgolide B as preventive treatment in a group of young patients suffering from migraine without aura.

\section{Patients and methods}

A small sample of 24 young patients suffering from migraine without aura entered the open-label prospective trial. Migraine without aura was diagnosed according to International Headache Society (IHS) criteria [7]. All patients were recruited at the Headache Center of C. Besta Neurological Institute. Inclusion criteria were: age between 8 and 18 years, initial onset of migraine at least one year before, and at least four migraine attacks (4 days/headache/ month) each of the 3 months prior to the screening. Exclusion criteria were: neurological or psychiatric diseases, neuroleptic or antidepressive medication within 6 months before screening, intake of prophylactic medication for migraine in the 6 months before screening, and medication overuse.

They were treated with a combination of Ginkgolide B $80 \mathrm{mg}$, coenzyme Q10 $20 \mathrm{mg}$, vitamin B2 $1.6 \mathrm{mg}$, and magnesium $300 \mathrm{mg}$ in oral administration twice per day, in the morning and in the evening, with meals, for 3 months. Number, duration, and severity of migraine attacks and analgesic intake were assessed in a diary card 1 month before the starting of the trial and during the treatment period. After 3 months, all patients were checked with their daily card for number, duration, severity headache episodes, and analgesic consumption. Follow-up sessions were planned for 3 , and 6 months after screening visit.

\section{Results}

This study includes a total of 24 patients (12 females and 12 males; mean age was $13.4 \pm 2.1$ ). Mean duration of illness was $3.7 \pm 2.6$ years. The mean number of days of headache per month was $9.3 \pm 7.6$; the mean number of medications per month was $5.7 \pm 4.4$.
As much as 14 patients (58.3\%) have already achieved the 6-month follow-up. The number of monthly migraine attacks was substantially reduced after 3 months of treatment with Ginkgolide B in relation to prestudy baseline. Starting with a mean baseline of $7.4 \pm 5$ attacks, clinical improvement was significant: the mean number of days of headache per month decreased to $2.2 \pm 2.8(p=0.0015)$, with a decrease of number of analgesics used for the attacks from $5.9 \pm 5.3$ to $1.5 \pm 2.2(p=0.013)$.

The treatment was well tolerated and the compliance was good: patients (and parents too) reported substantial improvement of their migraine compared to the situation prior to the study. None of the patients reported worsening of migraine.

\section{Conclusion}

The authors are aware that the uncontrolled open-label design of this study and the small sample of patients do not allow drawing definite conclusions regarding efficacy and tolerability of this kind of treatment. These preliminary data show that Ginkgolide B seems to be effective as preventive treatment in reducing migraine attack frequency and in attenuating the use of symptomatic medication in our small series of children with primary headache.

How Ginkgolide B improved migraine in young age is still unclear; to date the mechanism of action of Ginkgolide $\mathrm{B}$ on the CNS is not completely understood. It is believed that the main therapeutical effect may be due to the modulation and/or reducing the excitatory effect of glutamate in the CNS, and glutamate is involved in spreading depression [8]. Another effect of Ginkgolide B is to hinder the pathological action of PAF, that during some physiopathological circumstances in CNS, sensitizes the trigeminalvascular endings and induces pain [9].

Although the results are very preliminary, this treatment could be a good option for patients suffering from migraine without aura in particular for young patients, where therapies without side effects are needed.

Conflict of interest statement The authors declare that they have no conflict of interest related to the publication of this article.

Open Access This article is distributed under the terms of the Creative Commons Attribution Noncommercial License which permits any noncommercial use, distribution, and reproduction in any medium, provided the original author(s) and source are credited.

\section{References}

1. Bille B (1981) Migraine in childhood and its prognosis. Cephalalgia $1: 71-75$ 
2. Powers SW, Patton SR, Hommel KA, Hershey AD (2003) Quality of life in childhood migraines: clinical impact and comparison to other chronic illnesses. Pediatrics 112:e1-e5

3. Abu-Arefeh I, Russel G (1994) Prevalence of headache and migraine in schoolchildren. BMJ 309:765-769

4. Grazzi L, Andrasik F, Usai S, Bussone G (2007) Magnesium as a preventive treatment for paediatric episodic tension-type headache: results at 1-year follow-up. Neurol Sci 28(3):148-150

5. Andrasik F, Grazzi L, Usai S, Bussone G (2007) Pharmacological treatment compared to behavioural treatment for juvenile tensiontype headache: results at two-year follow-up. Neurol Sci 28(Suppl2): S235-S238

6. D'Andrea G, Bussone G, Allais G, Aguggia M, D'Onofrio F, Maggio M, Moschiano F, Saracco MG, Terzi MG, Petretta V,
Benedetto C (2009) Efficacy of Ginkgolide B in the prophylaxis of migraine with aura. Neurol Sci 30(Suppl 1):S121-S124

7. Headache Classification Subcommittee of the International Headache Society (2004) The international classification of headache disorders. Cephalalgia 24(suppl 1):1-152

8. Bryn W, Coran MH, Coran MH, Schultz PG, Rimbach G, Krucker $T$ (2004) Age-related effect of Ginkgo Biloba on synaptic plasticity and excitability. Neurobiol Aging 25:955-962

9. Nogami K, Hirashima Y, Endo S, Takaku A (1997) Involvement of platelet-activating factor (PAF) in glutamate neurotoxicity in rat neuronal cultures. Brain Res 754:72-78 